\title{
External Ventricular Drainage Infections Rates: Clinic Experiences
}

\author{
Serdar Çevik ${ }^{*}$, Celaleddin Soyalp², Enes Akkaya3 ${ }^{3}$, Serkan Kitis ${ }^{3}$, Hakan Hanımoğlu ${ }^{4}$ \\ ${ }^{1}$ Department of Neurosurgery, Agri State Hospital, Agrı, Turkey \\ ${ }^{2}$ Department of Anaesthesiology and Reanimation, Agri State Hospital, Agrı, Turkey \\ ${ }^{3}$ Department of Neurosurgery, Bezmialem University School of Medicine, İstanbul, Turkey \\ ${ }^{4}$ Department of Neurosurgery, Bilim University School of Medicine, İstanbul, Turkey \\ Email: “dr.serdarcevik@gmail.com,c.soyalp@hotmail.com, enesakkaya@yahoo.com, serkankitis@gmail.com, \\ drhakanhanimoglu@hotmail.com
}

Received 24 December 2015; accepted 26 January 2016; published 29 January 2016

Copyright (C) 2016 by authors and Scientific Research Publishing Inc.

This work is licensed under the Creative Commons Attribution International License (CC BY).

http://creativecommons.org/licenses/by/4.0/

(c) (i) Open Access

\section{Abstract}

In this study, we aimed to evaluate patients who develop ventriculostomy requirements and to evaluate the external ventricular drainage infection rates retrospectively at our clinic. In the study, dates between January 2012 and 2014, patients who were inserted external ventricular drainage with different indications were examined retrospectively. By using the medical record system, patients' demographics (age, sex), diagnostic, ventriculostomy indications and ventriculostomy time had been reached. By accessing laboratory data, the patients' cultures of cerebrospinal fluids biochemical tests were analyzed retrospectively. Within the period of the study, 20 in 117 patients with external ventricular drainage were not included the study because of shunt infection and shunt occlusion of extracted patients. During the treatment period 148 EVD were inserted to 97 patients. The number of male patients was 53; the number of female patients was 44 . When the reason of the examined patients' using external ventricular drainage was analyzed, $55 \%$ hemorrhagic cerebrovascular diseases, (subarachnoid hemorrhage, intraventricular hemorrhage, intracerebral hemorrhage, cerebellar hemorrhage, traumatic intracranial injury), $24.7 \%$ tumor induced use, $7.4 \%$ central nervous system infections (menengitis, apse), and $12.4 \%$ occlusive cerebrovascular diseases (hydrocephalus or brain edema that were developed after the infarct) were seen. During the course of our study, $23 \%$ of the surveyed 97 patients had leukocytosis. CFT culture of 12 patients found positive. In CFT cultures mostly coagulase-negative staphylococcus growth took place. Eventually, when we compare our infection proportions to the international literature, very large differences were not observed. Except for revisioned patients, no other criteria were found that increased the rate of infection. We think that a rigorous pre-operative preparation and a regular maintenance of external ventricular drainage may reduce the rate of infection.

\footnotetext{
${ }^{*}$ Corresponding author.
} 


\section{Keywords}

\section{External Ventricular Drainage, Infection, Ventriculostomy, Hydrocephalus}

\section{Introduction}

External ventricular drainage (EVD) is one of the neurosurgery methods frequently used by Brain surgeons. EVD is a surgical procedure that allows the establishment of a connection between cerebral ventricular system and external environment. EVD can be considered as a complementary venture for many neurosurgical enterprises such as intracranial hemorrhage, intracranial tumor, traumatic brain injury, cerebral edema and intracranial pressure measurement. However, as all surgery enterprises during and after the ventriculostomy process some complications may occur. Infection is the most important of these complications. Ventriculostomy connected infection rate is reported $0 \%-22 \%$ [1] [2]. After the ventriculostomy, so many studies were made for the cause of infection but a common cause has not been found [3]-[8].

In this study, we aimed to evaluate patients who develop ventriculostomy requirements retrospectively and to evaluate their infection rates at our clinic for 2 years.

\section{Materials and Methods}

In this retrospective study, all ventriculostomy procedures were examined dates between January 2012 and 2014.

When the patient information and operations searched, we received permission from the patients and their families and we used Bizmed (data system of our hospital). All ventriculostomy procedures, dates between January 2012 and 2014, were scanned in the system of Bizmed (health care system). EVDs that were inserted after the shunt removal because of shunt infection were not included in the study.

By using the medical record system, patients' demographic (age, sex), diagnostic, ventriculostomy indications and ventriculostomy time had been reached. By accessing laboratory data, the patients' cultures of cerebrospinal CFTs and biochemical tests were analyzed retrospectively.

We compared our results (ventriculostomy time, revision rate, culture of CFT, infection rates) with published international studies.

Written consent was provided from all of the patients before the surgical intervention and the patients were informed about possible involvement in a scientific study.

The statistical analysis was performed using SPSS 22.0. Median, minimum and maximum values were used as definitive statistical values. The analysis of contingency tables was performed with Fisher's exact test. Statistical significance was accepted as $\mathrm{P}<0.05$.

\section{Results}

20 in 117 patients with external ventricular drainage were not included the study because of shunt infection and shunt occlusion of extracted patients. 148 EVD were inserted to 97 patients during their treatment period.

All ventriculostomies were performed in the operating rooms according to the procedure defined below:

Under local anesthesia, following proper skin preparation right kocher point was identified. (The intervention was performed from the left kocher point in patients with right frontoparietal paranchymal hemorrhage) A $4 \mathrm{~cm}$ incision was made with no 21 scalpel. Periosteum was laterally dissected from the bone. A burr hole was placed with a high speed drill. Dura was cauteried with the bipolar cautery and a $5 \mathrm{~mm}$ incision was made with no 15 scalpel. Ventricular catheter was placed into the frontal horn. With the establishment of CSF drainage, the subcutaneous tissue and the skin were properly closed.

Evd catheter that contains antibiotic was not used in any of the patients. Before the surgical incision $1 \mathrm{~g}$ sefazolin was made all the preoperatively patients. Intravenous ceftriaxone vancomycin $3 \times 2 \mathrm{~g}$ and vancomycin $4 \times$ $500 \mathrm{mg}$ therapy were given to the 7 patients with a diagnosis of central nervous system infection (menengitis, abscess) from the moment of diagnosis to the duration of hospital stay.

The patients were followed at the intensive care unit or neurosurgery clinic from the moment of inserting 
EVD to the removing it by paying attention to sterilization. 3 times in a week, with using aseptic techniques samples of CFTs were taken by brain surgery doctors. Samples were delivered to microbiology laboratory within 10 minutes. EVDs that were not the requirement of the patients' EVDs were filmed aseptically and skin incision was sutured aseptically.

\subsection{Sex and Age}

53 patients in the study was male and 44 ones was female. The average age of the patients was 42 - 24 (Standard deviation (SD) \pm 25.81 ). The average age was 49. Our oldest patient that was mounted EVD was 88 years old. Our 2 patients' age was under 1 year and the youngest one was 3 days old. Maximum between 60 - 69 age group patients were inserted EVD and large propotion of our patients was composed of patients between the age of 0 9 and $40-69$.

\subsection{Indications for EVD}

The majority of the cases we used EVD (55.7\%) was due to hemorrhagic cerebrovascular diseases (subarachnoid hemorrhage, intraventricular hemorrhage, intracerebral hemorrhage, cerebellar hemorrhage, traumatic intracranial injury). Tumors were the second most common cause we used EVD (24.7\%). In this group we included tumor-caused obstructive hydrocephalus and post surgery EVD use for ICP monitorization patients. Other important indications are CNS infections (7.4\%) (meningitis, abcess), obstructive cerebrovascular disorders (12.4\%) (post-infarction hydrocephalus or cerebral odema) (Table 1).

\subsection{EVD Period}

We defined this period from applying EVD catheter till the need for EVD passed and to the point where we removed the EVD. We changed the EVD catheter at 14th day in patients with need of EVD more than 14 days. The average time for EVD application was 13.96 days (Table 2).

\subsection{CSF Culture}

Cerebral spinal fluid (CSF) samples were obtained from EVD catheter using aseptic technique. CSF samples were obtained in case of the changes of fever, white blood cell number and mental status were observed and they were sent to the microbiology laboratory for evaluation. CSF samples were sent to biochemistry laboratory in order to determine cell number and typing at the same time within 3 day intervals. During our study, 23 patients (23.7\%) developed leukocytosis in 97 evaluated patients (leukocytosis > 10 cells in 100 magnification). Coagulase-negative staphylococci is the most growth in 12 patients' culture who had positive CSF culture.

\begin{tabular}{ccc|}
\hline Table 1. Indications of EVD. & & \\
\hline Indications for EVD & No. of cases & $\begin{array}{c}\text { Percentage of } \\
\text { total }\end{array}$ \\
\hline Hemorrhage & 54 & 55.7 \\
Tumor & 24 & 24.7 \\
Infection & 7 & 7.4 \\
$\begin{array}{c}\text { Others (infarct, normal peresure } \\
\text { hydrocephalus, brain edema) }\end{array}$ & 12 & 12.4 \\
\hline
\end{tabular}

Table 2. Duration of EVD.

\begin{tabular}{cc}
\hline Duration of EVD & Days \\
\hline Mean & $13.96(\mathrm{SD}=14.439)$ \\
Longest & 91 \\
Shortest & 1 \\
\hline
\end{tabular}


Much growth of staphylococcus epidermidis in culture samples that suggests the possibility of transmission. Other pathogens are shown in the following table (Table 3).

\subsection{EVD Revision and Infection}

Revision was performed in case of situations like EVD occlusion or exceeding 14 days EVD requirements during the treatment of the 30 patients. Leukocytes were observed in 14 of the patients who underwent revision. Leukocytes were observed in only 6 patients who did not undergo revision (Fisher's exact test $P=0.000$ ). The observed rate of leukocytes in patients who underwent revision was statistically regarded significant compared to the patients who did not undergo revision. In 7 of the 30 patients who underwent revision bacteria were detected in their CSF culture. Bacterial growth occurred only in 5 of the 67 patients and from them, 3 patients' bacterial growth was coagulase-negative staphylococci. Bacterial growth was regarded significant in patients who underwent revision with the Fisher's exact test $(\mathrm{P}=0.043)$.

\subsection{Bleeding and Infections}

EVD was inserted to the 54 in 97 patients because of hemorrhagic cerebrovascular disease. Bacterial growth occurred in 6 among 54 patients. Among these 6 patients, 3 were inserted SAK and the other 3 patients were inserted EVD because of intracerebral hematoma. Because of bleeding EVD inserted patients' infection rate when compared considering other results, it was regarded statistically insignificant (Fisher's Exact test $\mathrm{P}=0.761$ ) (Table 4).

\section{Discussion}

Bacterial growth was $12.7 \%$ in two years follow-up of patients inserted-EVD (12/97). This infection rate is found to be close to the lower limit in comparison with the literatüre. The average infection rate in a literature study by Lozier and his friends in 2002 was reported to be $8.8 \%(2.13 \%-21.95 \%)$ [6]. But the infection rates associated with EVD were evaluated in most of these performed studies. The EVDs which have already inserted due to diagnosed meningitis were not assessed.

In our study, infection rates drop $10 \%$ if we exclude meningitis patients. When excluded our patients with the diagnosis of meningitis before, coagulase staphylococcus bacteria occurred in 4 patients among 9 who had bacterial growth in their CFT. In line with these results if we give more importance to the sterilization and EVD

\begin{tabular}{|cc|}
\hline Table 3. The distribution of bacteria in the CSF culture. \\
\hline CSF culture & No. of cases \\
\hline No growth & 85 \\
Total CSF positive culture & 12 \\
Coagulase negative staphylococcus & 4 \\
Klebsiella & 3 \\
Acinotobacter baumani & 2 \\
Pseudomonas argineusa & 2 \\
MRSA & 1 \\
\hline
\end{tabular}

Table 4. EVD infections rates caused by hemorrhage versus others.

\begin{tabular}{cccc}
\hline $\begin{array}{c}\text { Hemorrhage (infection } \\
\text { vs no infection) }\end{array}$ & $\begin{array}{c}\text { Hemorrhage as primary } \\
\text { presentation }\end{array}$ & $\begin{array}{c}\text { Hemorrhage not as } \\
\text { primary presentation }\end{array}$ & Total \\
\hline Infection & 6 & 6 & 12 \\
No infection & 48 & 37 & 85 \\
Total & 54 & 43 & 97 \\
\hline
\end{tabular}


care that we can prevent situations that may occur as a result of skin infections. So that our infection rates will fall to very low levels.

\section{Conclusion}

In conclusion, it does not show huge differences when we compare our infection rates with international literature. Except for revision patients another criterion was not found that significantly increases the rate of infection. We conclude that a rigorous peri-operative preparation and care of regular EVD may reduce the rate of infection.

\section{References}

[1] Bader, M.K., Littlejohns, L. and Palmer, S. (1995) Ventriculostomy and Intra Cranial Pressure Monitoring: In Search of a 0\% Infection Rate. Heart \& Lung, 24, 166-172. http://dx.doi.org/10.1016/S0147-9563(05)80012-3

[2] Muralidharan, R. (2015) External Ventricular Drains: Management and Complications. Surgical Neurology International, 25, 271-274. http://dx.doi.org/10.4103/2152-7806.157620

[3] Arabi, Y., Memish, Z.A., Balkhy, H.H., Francis, C., Ferayan, A., Al Shimemeri, A., et al. (2005) Ventriculostomy Associated Infections: Incidence and Risk Factors. American Journal of Infection Control, 33, 137-143. http://dx.doi.org/10.1016/j.ajic.2004.11.008

[4] Chi, H., Chang, K.-Y., Chang, H.C., Chiu, N.C. and Huang, F.Y. (2010) Infections Associated with Indwelling Ventriculostomy Catheter in a Teaching Hospital. International Journal of Infectious Diseases, 14, e216-e219. http://dx.doi.org/10.1016/j.ijid.2009.04.006

[5] Hoefnagel, D., Dammers, R., Ter Laak-Poort, M.P. and Avezaat, C.J. (2008) Risk Factors for Infections Related to External Ventricular Drainage. Acta Neurochirurgica (Wien), 150, 209-214. http://dx.doi.org/10.1007/s00701-007-1458-9

[6] Lozier, A.P., Sciacca, R.R., Romagnoli, M.F. and Connolly Jr., E.S. (2002) Ventriculostomy-Related Infections: A Critical Review of the Literature. Neurosurgery, 51, 170-181. http://dx.doi.org/10.1097/00006123-200207000-00024

[7] Martinez, E., Rello, J. and Coll, P. (1994) Clinicaldiagnosis of Ventriculostomy-Related Infections. Lancet, 344, 10151016. http://dx.doi.org/10.1016/S0140-6736(94)91671-3

[8] Park, P., Garton, H.J., Kocan, M.J. and Thompson, B.G. (2004) Risk of Infection with Prolonged Ventricular Catheterization. Neurosurgery, 55, 594-599. http://dx.doi.org/10.1227/01.NEU.0000134289.04500.EE 